\title{
Consensus on Recording of Gas Permeable Contact Lens Fit
}

\author{
James S Wolffsohn' ${ }^{1}$ Eef van der Worp ${ }^{2}$ John de Brabander ${ }^{3}$ \\ and the GP Consensus Group
}

1 Ophthalmic Research Group, Life and Health Sciences, Aston University, United Kingdom BSc MBA PhD FAAO FBCLA

2 University of Maastricht, Netherlands BSc PhD FAAO FBCLA

3 University of Maastricht, Netherlands MSc PhD FAAO 


\section{Abstract}

Purpose: To develop a new schematic scheme for efficiently recording the key parameters of gas permeable contact lens (GP) fits based on current consensus.

Methods: Over 100 established GP fitters and educators met to discuss the parameters proposed in educational material for evaluating GP fit and concluded on the key parameters that should be recorded. The accuracy and variability of evaluating the fluorescein pattern of GP fit was determined by having 35 experienced contact lens practitioners from across the world, grading 5 images of a range of fits and the topographer simulation of the same fits in random order using the proposed scheme. The accuracy of the grading was compared to objective image analysis of the fluorescein intensity of the same images.

Results: The key information to record to adequately describe the fit of an GP was agreed as: the manufacturer, brand and lens parameters; settling time; comfort on a 5 point scale; centration; movement on blink on a \pm 2 scale; and the Primary Fluorescein Pattern in the central, mid-peripheral and edge regions of the lens averaged along the horizontal and vertical lens axes, on a \pm 2 scale. On average $50-60 \%$ of practitioners selected the median grade when subjectively rating fluorescein intensity and this was correlated to objective quantification $(\mathrm{r}=0.602, \mathrm{p}<0.001)$. Objective grading suggesting horizontal median fluorescein intensity was generally symmetrical, as was the vertical meridian, but this was not the case for subjective grading. Simulated fluorescein patterns were subjectively and objectively graded as being less intense than real photographs $(\mathrm{p}<0.01)$.

Conclusion: GP fit recording can be standardised and simplified to enhance GP practice. Keywords: rigid gas permeable contact lenses (RGP); fitting characteristics; lens evaluation; record keeping; fluorescein pattern observation 


\section{Introduction}

Accurate record keeping when fitting contact lenses is essential for medico-legal protection as well as to allow informed refitting decisions to be made in the future. This is particularly important if follow-up care is undertaken by a different eye care practitioner. While soft lenses fit evaluation has been analysed objectively, and a simplified but comprehensive schematic for recording the findings has been developed, ${ }^{1}$ no such research has been published on gas permeable contact lenses (GPs).

Unlike soft lenses which largely conform to the contour of the ocular surface, GP fit can be evaluated by the pattern of fluorescein under the lens. Evaluation of the lens fit with fluorescein has been used ever since rigid lenses came on to the market in the 1950s. It has been proven to be very useful in clinical practice to identify where the lens comes on contact with the corneal surface (termed 'touch'), although the human eye is only able to detect fluorescein layers with a thickness of at least $20 \mu \mathrm{m} .{ }^{2,3}$ This results in tear layers thinner than $20 \mu \mathrm{m}$ appear dark. For this reason for instance, it is difficult to fit orthokeratology lenses relying on fluorescein patterns alone. ${ }^{4}$

Suboptimal lens fit has been proven to be of influence on comfort of GP lens wear. In a study at the University of Maastricht it was found that comfort improved over a period of three months in the optimal lens fit group, but not in the sub-optimal lens fit group. The difference after three months between these two groups was 2 on a 10 point scale; a statistically and most probably clinically significant difference. ${ }^{5}$

One of the few complications that is typical for, and exclusively seen in, GP lens wear is 3- 
and 9-o'clock staining. The prevalence of 3- and 9-o'clock staining is reported to be up to $80 \%$, of which $10-15 \%$ is estimated to be of clinical significance. In a review paper, five out of the total of eleven treatment options to decrease or remedy 3- and 9-o'clock staining identified in the academic literature were related to lens parameters, three to lens performance. ${ }^{6}$ Hence improving lens fit, by following the shape of the cornea more closely, could remedy the condition of 3- and 9-o'clock staining.7

Corneal topography could be beneficial in this process, but fluorescein evaluation is crucial in the final judgment of the lens fit. In addition, suboptimal GP lens fit leads to corneal warpage. ${ }^{8-10}$ The mean recovery time for corneal warpage in GP lens wearers (as assessed by a topography change less than $0.5 \mathrm{D}$ ) is $8.8 \pm 6.8$ weeks. ${ }^{12}$ The resting position of the lens on the cornea seems to play an important role; the topography of warped corneas is usually characterised by a relative flattening of the cornea underlying the GP contact lens in its resting position. Lenses that ride high, for example, produce flattening superiorly and result in a relatively steeper contour inferiorly. ${ }^{11}$

As well as the fluorescein pattern, other aspects of GP fit have also been proposed to be important to optimised fitting such as centration and coverage, lid attachment and surface wettability. ${ }^{13-16}$ However, there is little evidence in the academic literature as to which of these parameters independently contribute to comfortable, healthy GP lens wear. Unlike soft lenses, 17,18 there are no studies modelling how lens design, material and anterior eye parameters influence lens movement. 
An added level of complexity results from the dynamic nature of an GP fit, with the movement on blink typically an order of magnitude larger than that of a soft contact lens. Movement of a lens is critical to provide sufficient oxygen exchange over the corneal surface as well as to remove trapped debris, inflammatory cells, and other tear components that would otherwise accumulate under the lens. ${ }^{19}$ The tear layer between the contact lens and cornea also reduce the friction between the surfaces, avoiding significant mechanical interaction. While soft lenses with limited mobility have been shown to have a more negative impact on ocular physiology than well fitting soft lenses, ${ }^{20}$ this is not well researched for GPs. This is probably because tear exchange beneath an GP is typically 15$16 \%$ compared to $1-2 \%$ for soft lenses, ${ }^{19,21}$ although tear mixing with differing amounts of lens movement does not seem to have been examined.

Therefore, to assess all these parameters takes a significant amount of time. Time pressures of clinical practice and a lack of consensus as to the key parameters that need to be optimised to achieve an appropriate GP fit results in varied and sometimes minimal documentation of GP fit characteristics. Notations such as "good" or "adequate" can be highly subjective and of limited use in future patient aftercare. This is especially the case in large practices with multiple eye care specialists, or when communicating with colleagues or lens manufacturer/consultants. Therefore, the relative importance of contact lens fit characteristics should be decided upon to develop a time efficient, but sufficiently detailed, description of lens performance for recording in clinical practice.

This study aimed to gain consensus from GP practitioners across the world as to the key parameters that should be observed and recorded and characterized to characterize 
corneal GP contact lens fit. In addition, practitioner's ability to subjectively scale fluorescein patterns was assessed against objective image analysis. This allowed for a simplified method of recording the GP lens fit of trialed lenses in clinical practice to be devised. 


\section{Method}

Over one hundred established GP fitters and educators met to discuss the parameters proposed in educational material for evaluating GP fit and concluded on the key parameters that should be recorded. These focus groups were held in 2011-13 at the British Contact Lens Association Conference, Netherlands Contact Lens Conference and British Committee of Contact Lens Educators (BUCCLE). In addition, members of the International Association of Contact Lens Educators (IACLE) and the Association of Optometric Contact Lens Educators (AOCLE) were contacted for their opinions and comment.

The focus groups were facilitated by the authors to discuss all elements that contribute to the GP fit decision making process and to debate over those elements that are critical to the decision to modify lens parameters. It was emphasised that while making clinical decisions cannot be prescriptive on a limited range of findings and the GP fitted should be based on clinical interpretation and experience, the recording of the key parameters could be standardised and terminology emotive of clinical decisions, such as "excessive", were hence avoided. Observation should also be totally independent of clinical consequences, as something isn't necessarily 'worse' because a different treatment plan is required.

The accuracy and variability of evaluating the fluorescein pattern of central the GP fit using the scheme proposed and agreed on by the consensus groups was determined by 35 experienced GP practitioners from around the world using five photographic images captured through a yellow filter chosen to represent a range of steep-to-flat and sphericalto-toric fits and the five topographer simulations of the same fits with the Medmont E300 
corneal topographyer (Medmont Ltd, Melbourne, Australia). These were presented in random order and the practitioners were not aware that the topographer simulations were paired with photographs of lens fits to avoid bias in their grading. The graded regions of the photographs and simulations were objectively analysed using bespoke Labview Vision software (National Instruments, Austin, Texas, USA) using the 256 point 8 bit) intensity scale for green light for comparison with the average practitioner grading. Concordance was assessed between practitioners as to the percentage selecting the median grade for each corneal zone. The average grade was correlated to the image analysis objectively measured green intensity across all zones and meridians using Spearman's rank correlation. 


\section{Results}

It was agreed in the workshop that the following should be recorded when fitting or checking an GP contact lens fit:

- Lens specifications including the lens design and manufacturer, base curves and associated diameters and lens power (as well as centre thickness, edge lift design, material, colour and any special requests such as prism, truncation or lenticularization if not previously recorded in the notes) Even if the patient was previously fitted in the practice, it is often not clear which lens the patient is wearing if it is not recorded along with the lens fit evaluation.

- The settling time in minutes, which should be sufficient for initial adaptation to have occurred, any induced tearing to have subsided and the lens to be comfortable. The consensus was that 20 minutes should meet these criteria in most cases. If this is not the case, the lens fit recorded will not be representative of the true fit, and if the lens does not fully settle, then it is unlikely the lens would be prescribed.

- Comfort on a 0 (poor) to 4 (perfect) scale. The 5 point range was determined to be sufficient to differentiate between comfort states, without scale grades being redundant. This matches familiar grading scale increments, although in reverse as asking a patient about any discomfort has the potential to negatively influence the patients reflection on the lens fitting experience.

- White diffuse light to evaluate:

o Centration - as the lens is mobile, the amount of decentration will change with time after each blink, so to make clinical decisions, centration would be noted as ' $\mathrm{L}$ ' to indicate the GP is crossing the limbus or ' $\mathrm{P}$ ' when the optic 
zone encroaches across the pupil in dim light (mesopic conditions), together with the direction; otherwise a 'C' could indicate adequate centration. Coverage was felt to be largely covered by centration indicators, with an ' $\mathrm{X}$ ' denoting the diameter is insufficient for the optic zone to encompass the pupil throughout the inter- blink period.

o Movement - the amount of movement on blink should be recorded on a -2 to +2 scale:

- $\quad+2$ : $\quad>2 \mathrm{~mm}$ movement

- +1 1.6 to $2.0 \mathrm{~mm}$ movement

- $\quad 0 \quad 1.0$ to $1.5 \mathrm{~mm}$ movement

- $-1 \quad 0.5$ to $0.9 \mathrm{~mm}$ movement

- $-2<0.5 \mathrm{~mm}$ movement

Fluorescein assessment - sodium fluorescein should be applied using a fluoret with excess saline removed 22 ideally to the temporal superior conjunctiva to maximise longevity on the ocular surface and the GP lens evaluated when centred, after a few blinks. A yellow filter should be used in front of the observation system to optimally view the fluorescein fluorescence. ${ }^{22}$ While some practitioners like to evaluate the lens in its resting position after a blink as well, the lens parameters are traditionally chosen to conform with the central cornea and if the lens is too far decentred from the central cornea, its parameters should be altered to improve centration. Hence the fluorescein pattern when the GP is centred (which can involve lid manipulation by the practitioner) should be recorded. The 'Primary Fluorescein Pattern' should be graded in the two principal meridians, recording the central (the inner $50 \%$ of the radius except the very centre where the two meridians cross), mid peripheral (the outer $50 \%$ of the radius except for the final edge curve) and 
edge zone (the final edge curve) fluorescein intensity (Figure 1). If the Primary Fluorescein Pattern shows clear oblique astigmatism, the fluorescein intensity should be graded along these meridians and a note made of the oblique angle under which the grading is conducted.

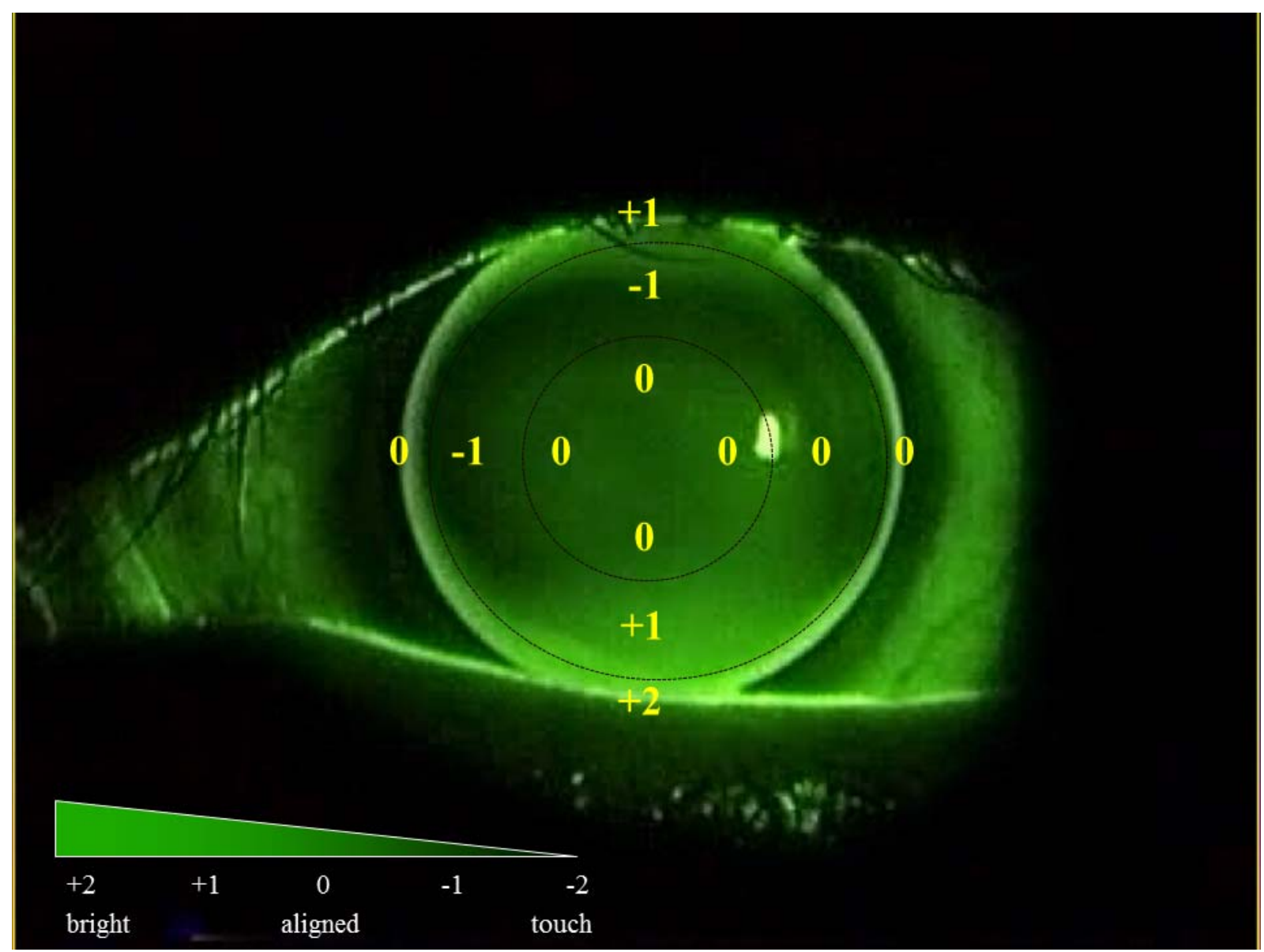

Figure 1: $\quad$ Fluorescein picture with overlaid central, mid-peripheral and edge zones, graded for fluorescein intensity on a +2 to -2 scale along the principal meridians (image courtesy of Ron Beerten, Procornea, The Netherlands).

Fluorescein intensity grading concordance was assessed between practitioners as to the percentage selecting the median grade for each corneal zone (i.e. the one chosen most commonly by the graders). For the inner ring concordance was $60 \pm 11 \%$ which was 
significantly greater than the mid-peripheral grading (48 $\pm 10 \% ; \mathrm{p}=0.036)$, but not significantly different compared to the edge zone (53 $\pm 10 \% ; p=0.248$; Figure 2 ). The average grade was correlated to the image analysis objectively measured green intensity across all zones and meridians using Spearman's rank correlation. There was a strong correlation between the average subjective grading and objective quantification of fluorescein intensity $(r=0.602, p<0.001)$.

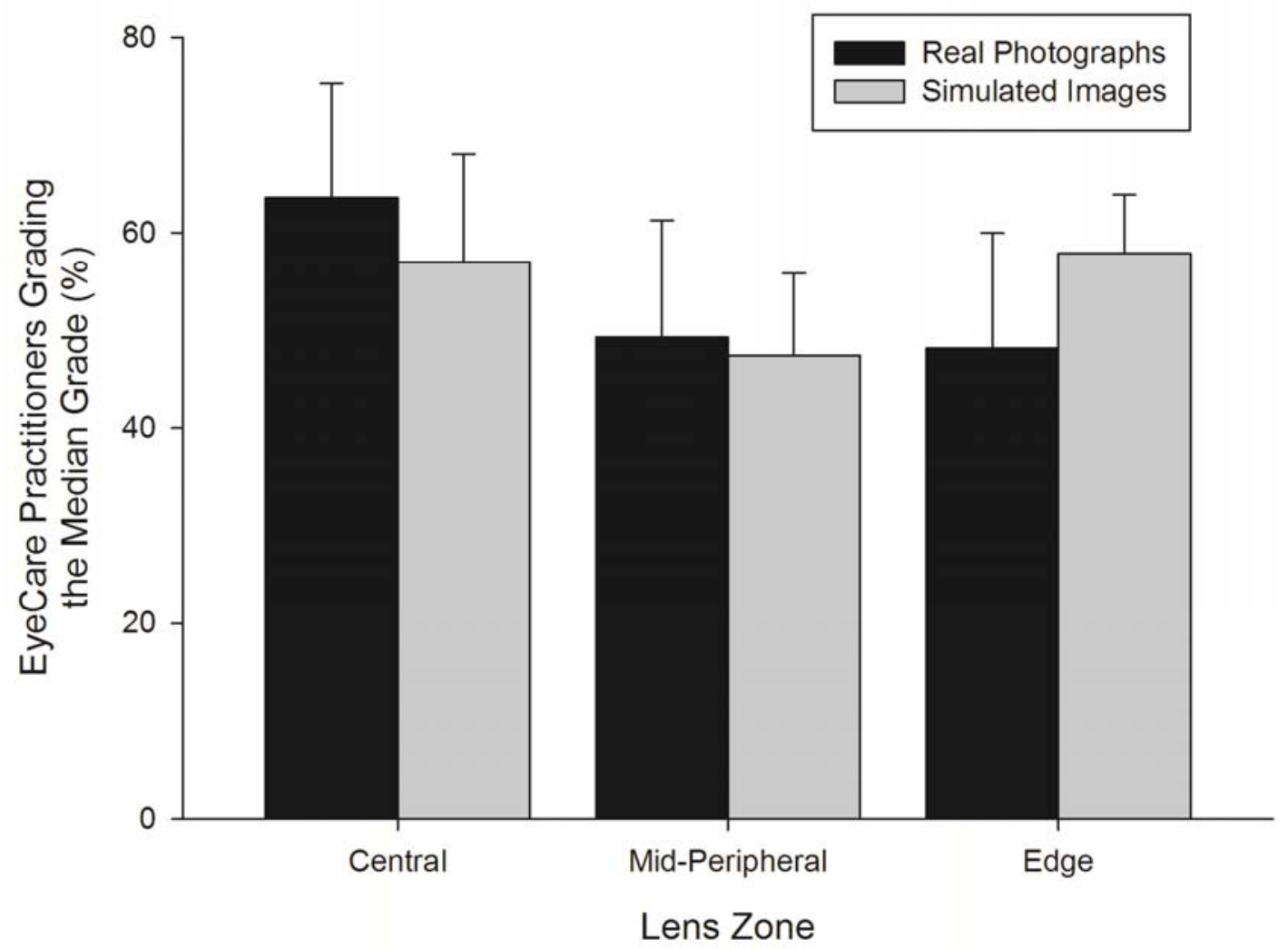

Figure 2: $\quad$ Concordance (eyecare practitioners selecting the median grade) in the central, mid-peripheral and edge lens zones for both real photographs and simulated images of fluorescein patterns. Error bars $=1$ S.D. $n=35$ practitioners and 5 averages of fluorescein pattern intensity grading on a +2 to -2 scale along the principal meridians. 
The subjective grading was not symmetrical between superior and inferior or between nasal and temporal for the inner $(\mathrm{F}=70.318, \mathrm{p}<0.001 ; \mathrm{F}=28.533, \mathrm{P}<0.001)$ or mid peripheral $(\mathrm{F}=22.831, \mathrm{p}<0.001 ; \mathrm{F}=14.969, \mathrm{P}<0.001)$ fluorescein intensity grading zones (respectively). However edge fluorescein intensity grading was symmetrical between superior and inferior $(\mathrm{F}=3.477, \mathrm{p}=0.071)$, although not between nasal and temporal $(\mathrm{F}=$ 36.869, $\mathrm{P}<0.001$ ) zones. Hence the fluorescein intensity grades in the steeper and flatter meridians generally differed on either side of the lens centre. Conversely, objective grading was not significantly different between superior and inferior $(F=1.256, p=0.292)$ or between nasal and temporal $(\mathrm{F}=0.833, \mathrm{p}=0.385)$ positions.

Simulated fluorescein patterns were subjectively graded significantly differently to real photographs (average grade $-0.3 \pm 1.3$ vs $0.2 \pm 1.2 ; \mathrm{F}=47.069, \mathrm{p}<0.001$ ), and there was an interaction with grading zone (inner $-0.1 \pm 1.3$ vs $0.6 \pm 1.0$; mid-peripheral $-0.2 \pm 1.2$ vs 0.2 \pm 1.2 ; edge $-0.5 \pm 1.5$ vs $-0.3 \pm 1.3 ; \mathrm{F}=5.950, \mathrm{p}=0.003$ ) and position on the GP (superior 0.5 \pm 1.1 vs $0.4 \pm 1.1$; temporal $-0.8 \pm 1.2$ vs $-0.1 \pm 1.3$; inferior $0.1 \pm 1.3$ vs $0.5 \pm 1.1$; nasal $-0.8 \pm$ 1.2 vs $-0.2 \pm 1.2 ; \mathrm{F}=27.178, \mathrm{p}<0.001$ ) respectively. The correlation between the objective quantification of the real and simulated fluorescein patterns was also only moderate $(\mathrm{r}=$ $0.256, \mathrm{p}=0.048)$, with the 'brightness' of the real photographs being greater $(40.2 \pm$ $17.2 \%)$ than the simulated images $(30.4 \pm 24.7 \%$; t-test $p=0.008)$. 


\section{Discussion}

This study set out to establish a consensus on the recording of GP lens fit. While grading is sometimes directly related to management, recording of GP fitting characteristics should be informative to the management process based on clinical judgment using all the available information, rather than dictate it. Based on clinical and anecdotal feedback, consensus was reached on the ideal settling time being 20 minutes; the rating of discomfort on a 5 point scale, which has previously been shown to sufficiently differentiate between comfort states, without scale grades being redundant ${ }^{1}$; white diffuse light evaluation of centration and coverage, with recording linked to issues that would affect clinical management such as crossing the limbus or the optic zone is encroaching across the pupil; and movement on blink which is critical to healthy ocular physiology. ${ }^{19,21}$

Fluorescein assessment was concluded to be best evaluated with the GP lens centred, using lid manipulation if necessary, using a \pm 2 grading system in the four principal meridians, recording the central, mid peripheral and edge zone fluorescein intensity. This 'Primary Fluorescein Pattern' evaluation was utilised by thirty-five experienced practitioners on both real and topographer simulated fluorescein images. Concordance between practitioners was moderate, with approximately 50-60\% agreeing on the same grade, although it was slightly worse for the mid-peripheral zone. This may be due to difficulties in judging the most appropriate position to grade the fluorescein intensity for this zone. The zones were arbitrarily chosen to be approximately $5 \mathrm{~mm}$ and $8 \mathrm{~mm}$ in diameter, but a graticule in the slit lamp eye-piece or the imaging software depicting these zones should assist the practitioner in enhancing their grading. The correlation with objective analysis of 
fluorescein intensity was also moderate, with subjective grading accounting for $36 \%$ of the variance.

While objective measurement of fluorescein intensity was symmetrical in the vertical and horizontal meridians; however, this was not generally the case for subjective grading. The intensity of the fluorescein was consistently graded as greater in the superior position compared to the nasal, and greater in the temporal position compared to the nasal, for each of the zones. This would suggest that the lack of symmetrical subjective fluorescein pattern grading may be due to off-axis clinical positioning of the slit-lamp illumination system rather than differences in fit. Hence it would seem appropriate to average the grading along the vertical median (so one grade for edge fluorescein intensity as the average at the top and bottom of the lens, one grade for mid-peripheral fluorescein intensity and one grade for central lens fluorescein intensity) and along the horizontal meridian to halve the number of fluorescein intensity grades to record.

Interestingly, simulated fluorescein patterns were subjectively graded as significantly less intense than actual photographs in each zone and this was confirmed objectively. In addition, the correlation between the objective quantification of the real and simulated fluorescein patterns was significant, but only just. Hence this would suggest that topographer simulation images could be made more intense to more closely depict clinical images when an GP of these parameters is fitted to the eye.

Hence, from this research the suggested schematic for recording GP fit is demonstrated in Figure 3 examples. While more could be recorded regarding GP lens fit particularly when 
fitting irregular corneas, this standardised schematic format is quick to notate and should be sufficient for most decision making, providing a substantial benefit over current practice.

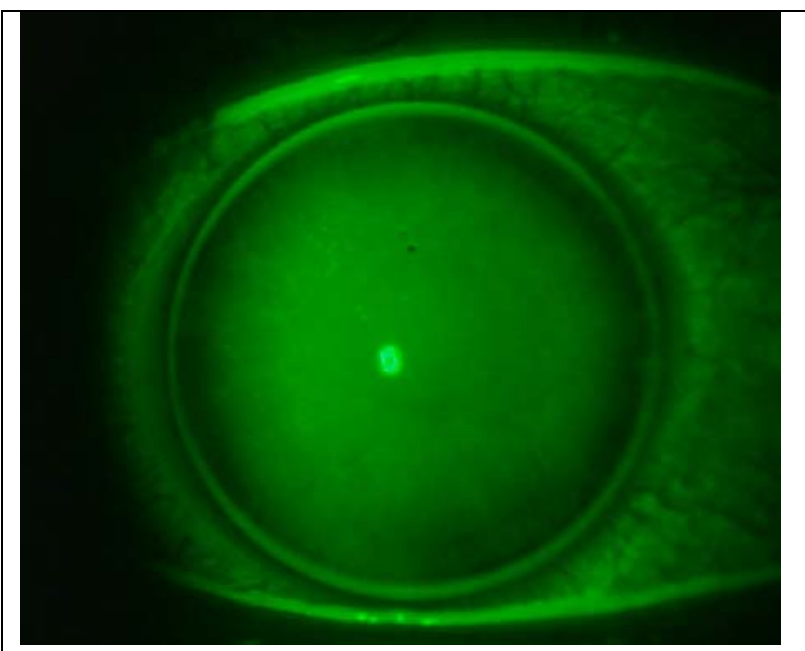

A) Manufacturer, Brand, 7.80/9.8+3.50

Settling time: 20 mins

Comfort:

Centration:

C

Movement on blink: -1

PFP; C M E

$\mathrm{V}:+2 \quad 0-1$

$\mathrm{H}:+2 \quad 0-1$

Figure 3A: $\quad$ Example of how to record a well centred, steep lens (fluorescein grading median of 35 experts)

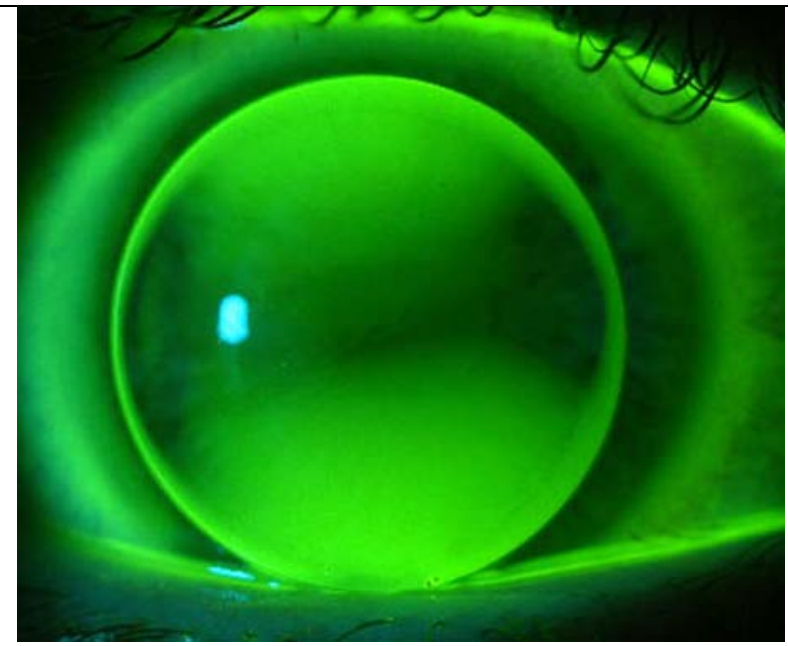

B) Manufacturer, Brand, 8.20/9.8 -2.50

Settling time: 20 mins

Comfort:

2

Centration:

L temporally

Movement on blink: +1

PFP; $\quad$ C M E

$\mathrm{V} 100^{0}:+1+2+2$

$\mathrm{H} 10^{0}:-1-2+1$

Figure 3B: Example of how to record a slightly oblique toric lens fit, which is mobile and crosses the limbus (fluorescein grading median of 35 experts) 


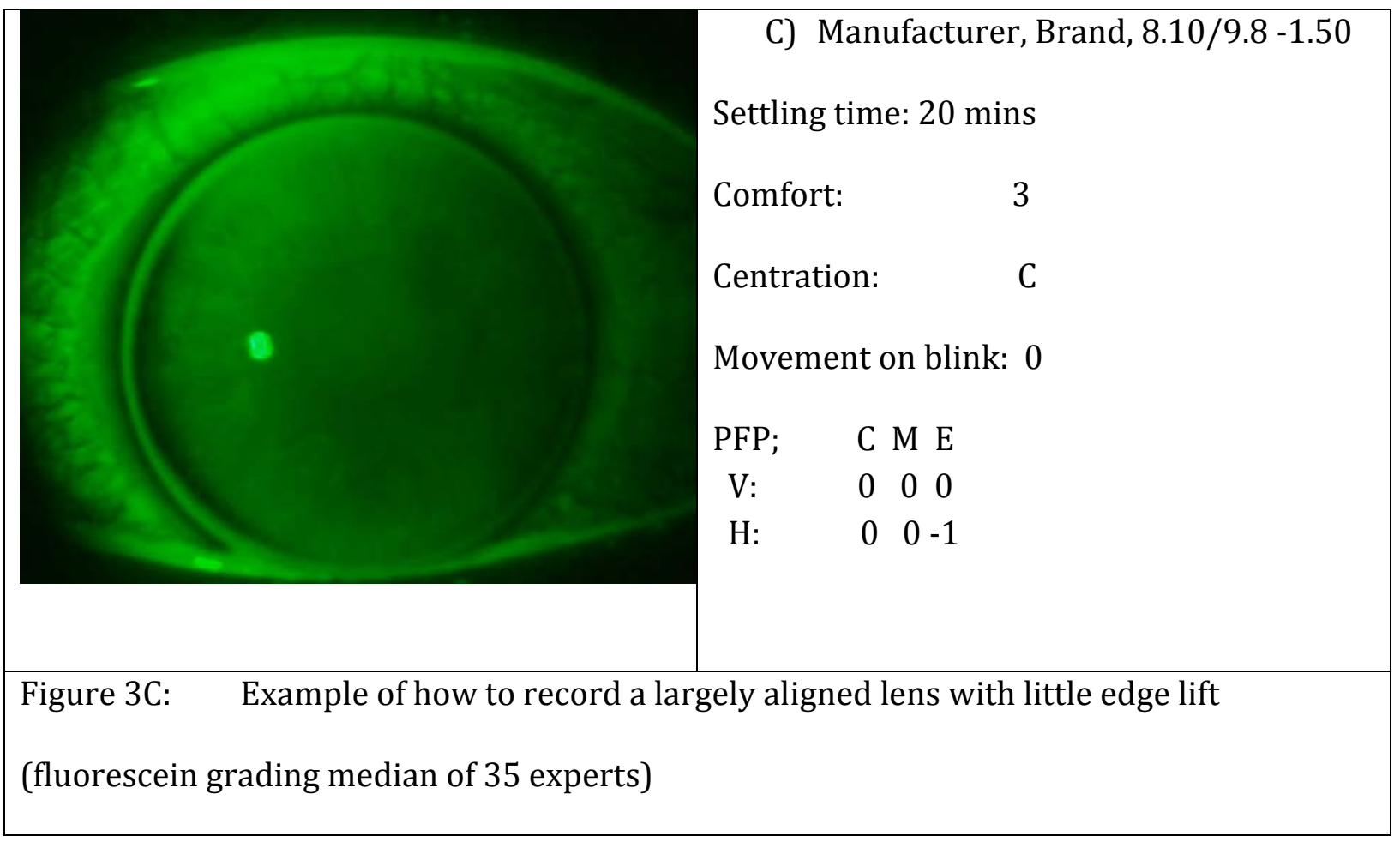

\section{Conflicts of Interest:}

None

\section{Acknowledgement:}

We are grateful to Patrick Caroline, FAAO, FCLSA, FIOS and Randy Kojima, FAAO, FSLS, FIOS of Pacific University College of Optometry for their provision of the images that were graded in this study and to the GP Lens Institute (www.GPLI.info) for their support.

\section{GP Consensus Group:}

Lens Arnham; Eddy Bakker; Ide Bangma, PAC Beks; Edward Bennett; J Bliekendaal; Wim Borst; K Brindha; H van Bruggen; N Buijs; Zaheer Cassim; Catharine Chisholm; Arnold Cochrane; Robert Conway; Caroline Christie; Jyoti Dave; M De Winter; R Dorst; CMA van Duijn; H Eek; Dean Dunning; Donald Egan; Reza van Etten; IT Evenhuis; Ian Forrest; Daniel Fuller; LD Gertzen; JJ van Gerven; SD van Gerven-Stoppelenburg; Jose M González-Méijome; PS Groen-Vegt; A Groet; Tony Harknett; Kathleen Helsen; Elien Janssen; A Kamst; NJ Kerst; 
Walter Kochen; Koen Koers; SA Kolbe; Peter S Kollbaum; W Kram; Ron de Lau, Wolfgang Laubenbacher; N Manessa; Andrew McLeod, Graham Mouat; Vicente S. Martín Montañez; Myrian T Mayorga; Nirav Mehta; Leon Meijboom; Cristian Mertz; M Mohr-Bakker; JA Mohunlol; Monique Mommen; Judith Morris; Marian Nagtzaam, Shehzad Naroo; Katherine Oliver; Dieuwke van Ooik; G Oosthuizen, J van Overbeek; MS Preeji; M Sas-Meertens; Jayne Schofield; PTA Schreven; Stefan Schwarz; Helmer Schweizer; CA Sikking- van Reenen; E Sissing; Alan Tomlinson; Marco Tovaglia; GCFE Vanschoenwinkel; César Villa-Collar; S Verdonk; J Verdoodt; Sandra Woldringh, Marisa Tesón Yudego. 


\section{References}

1 Wolffsohn JS, Hunt OA, Basra AK. Simplified recording of soft contact lens fit. Contact Lens Ant Eye 2009;32:37-42.

2 Carney LG. Luminance of fluorescein solutions. Am J Optom Arch Am Acad Optom 1972;49:200-4.

3 Guillon M, Sammons W. Contact lens design. In: Ruben M, Guillon M, ed. Contact Lens Practice. London: Chapman \& Hall, 1994:87-112.

$4 \quad$ Mountford J, Cho P, Chui WS. Is fluorescein pattern analysis a valid method of assessing the accuracy of reverse geometry lenses for orthokeratology? Clin Exp Optom 2005;88:33-8.

5 Van der Worp E, De Brabander J, Lubberman B, Marin G, Hendrikse F. Optimising RGP lens fitting in normal eyes using 3D topographic data. Contact Lens Ant eye 2002;25:959.

6 Van der Worp E, De Brabander J, Swarbrick H, Nuijts R, Hendrikse F. Corneal desiccation in rigid contact lens wear: 3- and 9-o'clock Staining - a review. Optom Vis Sci 2003;80:280-290.

$7 \quad$ Van der Worp E. Corneal desiccation in rigid contact lens wear: Time to deal with 3- and 9-o'clock Staining. PhD thesis, University of Maastricht (the Netherlands), Nov 2008.

8 Maeda N, Klyce SD, Hamano H. Alteration of corneal asphericity in rigid gas permeable contact lens induced warpage. CLAO J 1994;20:27-31.

9 Ruiz-Montenegro J, Mafra CH, Wilson SE, Jumper JM, Klyce SD, Mendelson EN. Corneal topographic alterations in normal contact lens wearers. Ophthalmology 1993;100:128-34. 
10 Wilson SE, Lin DT, Klyce SD, Reidy JJ, Insler MS. Topographic changes in contact lensinduced corneal warpage. Ophthalmology 1990;97:734-44.

11 Wilson SE, Lin DT, Klyce SD, Reidy JJ, Insler MS. Rigid contact lens decentration: a risk factor for corneal warpage. CLAO J 1990;16:177-82.

12 Wang X, McCulley JP, Bowman RW, Cavanagh HD. Time to resolution of contact lensinduced corneal warpage prior to refractive surgery. CLAO J 2002;28:169-71.

13 Phillips A. Rigid gas permeable and hard corneal lens fitting. In: Phillips A, Stone J, ed. Contact Lenses. London: Butterworth, 1989:333-81.

14 Ruben M, Guillon M. Contact Lens Practice. London: Chapman \& Hall, 1994.

15 Bennett E, Egan D. Rigid gas permeable lens problem solving. J Am Optom Assoc $1986 ; 57: 504-11$.

16 Dougal J. Abrasions secondary to contact lens wear. In: Tomlinson A, ed. Complications in Contact Lens Wear. St. Louis: Mosby, 1992:123-56.

17 Martin DK, Holden BA. Forces developed beneath hydrogel contact lenses due to squeeze pressure. Phys Med Biol 1986;30;635-49.

18 Bibby MM, Tomlinson A. A model to explain the effect of soft lens design specifications on movement. Am J Optom Physiol Opt 1983;60:287-91.

19 McNamara NA, Polse KA, Brand RJ, Graham AD, Chan JS, McKenney CD. Tear mixing under a soft lens: effects on lens diameter. Am J Ophthalmol 1999;127:659-65.

20 Young G, Coleman S. Poorly fitting soft lenses affect ocular integrity. CLAO J 2001;27:68-74.

21 Kok JHC, Boets EPM, van Best JA, Kijlstra A. Fluorophotometric assessment of tear turnover under rigid contact lenses. Cornea 1992;11:515-7. 
22 Peterson RC, Wolffsohn JS, Fowler CW. Optimization of Anterior Eye Fluorescein Viewing. Am J Ophthalmol 2006;142:572-5. 